\title{
Traveling waves of a diffusive epidemic model with nonlinear infection forces
}

\author{
Mingju Ma, Sanyang Liu and Jun Li*
}

"Correspondence:

lijun@xidian.edu.cn

School of Mathematics and

Statistics, Xidian University, Xi'an,

Shaanxi 710071, P.R. China

\begin{abstract}
We study an SIRS epidemic model with diffusion and nonlinear incidence rate is studied. In the presence of spatial diffusion, we show that the local stability and the global stability are completely determined by the basic reproduction ratio $R_{0}$. Under the so-called quasi-monotonicity assumption, we use the upper-lower solution method and Schauder's fixed point theorem to establish the existence of traveling wave and obtain an explicit expression of the minimum wave speed $c^{*}$. Numerical examples are given to confirm rich dynamical features of the model.
\end{abstract}

MSC: 34K10; 92D30

Keywords: epidemic model; stability; traveling wave

\section{Introduction}

Since the Kermack-McKendrick epidemic SIR model [1] was proposed in the 1920s, in which the total population is divided into three classes of susceptible individuals (S), infectious individuals (I), and recovered individuals (R), the SIR and the SIRS types of epidemic models have been studied by a great deal of researchers [2-5]. In most epidemic models, the SIR (acquired immunity is permanent) and SIRS (acquired immunity is nonpermanent) models always make the assumption that the total population is constant in size if the disease spreads quickly and dies out within a short time or the disease rarely causes deaths, which can make the mathematical analysis easier by reducing the model into a planar system. In modeling communicable diseases, one of the most important factors in producing rich dynamics is the incidence rate. Indeed, bilinear interaction incidence and standard incidence given by $\beta I S$ or $\frac{\beta I S}{N}$ are frequently used [6-10]. Such models always admit a globally asymptotically stable disease-free equilibrium or endemic equilibrium, corresponding to the disease-free steady state or endemic steady state. However, actual data and evidences observed for many diseases (such as influenza, measles, mumps, chickenpox) show that dynamics of disease transmission are not always as simple as shown in these models, and classical epidemic models also cannot explain lots of important phenomena, such as periodic oscillations [11] and so on.

Thus, in recent years, many scholars have taken into account oscillations caused by incidence rates and proposed various nonlinear incidence rates [12-19]. With these nonlinear incidence rates, many interesting and complicated transmission dynamics of epidemics have been shown. The nonlinear incidence rate $k I^{p} S^{q}(k, p, q>0)$ is investigated by Liu et

(c) $2016 \mathrm{Ma}$ et al. This article is distributed under the terms of the Creative Commons Attribution 4.0 International License (http://creativecommons.org/licenses/by/4.0/), which permits unrestricted use, distribution, and reproduction in any medium, provided you give appropriate credit to the original author(s) and the source, provide a link to the Creative Commons license, and indicate if changes were made. 
al. [17]. van den Driessche and Watmough [20, 21] also studied an incidence rate of the form $b I\left(1+m I^{k-1}\right) S$, where $b>0, m \geq 0$, and $k>0$. Capasso and Serio [16] introduced a saturated incidence rate $g(I) S=\frac{\beta I S}{1+\beta \delta I}(\delta>0)$ in an epidemic model when they studied the cholera epidemic in 1973. Derrick and van den Driessche [22] considered a generalized form of nonlinear incidence rate in an epidemic model. The effect of behavioral changes has been incorporated by Liu et al. [18] using the nonlinear incidence rate $\frac{k l}{1+\alpha I^{h}}$ with $k, l, \alpha, h>0$. Hu et al. [23] considered the same nonlinear incidence rate. Ruan and Wang [15] considered the fixed infection force $\frac{a I^{2}}{b+I^{2}}$, which corresponds to a saturated infection force, and obtained rich dynamical behaviors in an SIRS model.

Wang [19] considered an SIRS epidemic model with nonlinear incidence of general form,

$$
\left\{\begin{array}{l}
\frac{\mathrm{d} S}{\mathrm{~d} t}=d N-d S-\frac{a I}{f(I)} S+b R, \\
\frac{\mathrm{d} I}{\mathrm{~d} t}=\frac{a I}{f(I)} S-(d+\gamma) I, \\
\frac{\mathrm{d} R}{\mathrm{~d} t}=\gamma I-(d+b) R,
\end{array}\right.
$$

where $d$ is the birth rate and death rate of the population, $b$ is the rate of removed individuals who lose immunity and return to the susceptible class, $\gamma$ is the recovery rate of infective individuals, $\frac{1}{f(I)}$ represents the effect of intervention policies on the reduction of valid contact coefficient $a$, and $\frac{a I}{f(I)}$ is the infection force. He assumed that the population size is a constant $N=S+I+R$ and, to ensure a nonmonotonic infection force, that (i) $f(0)>0$ and $f^{\prime}(I)>0$ for $I>0$ and (ii) there exists $\xi>0$ such that $\left(\frac{I}{f(I)}\right)^{\prime}>0$ for $0<I<\xi$ and $\left(\frac{I}{f(I)}\right)^{\prime}<0$ for $I>\xi$. Then he obtained the less-dimensional model

$$
\left\{\begin{array}{l}
\frac{\mathrm{d} I}{\mathrm{~d} t}=\frac{a I}{f(I)}(N-I-R)-(d+\gamma) I, \\
\frac{\mathrm{d} R}{\mathrm{~d} t}=\gamma I-(d+b) R .
\end{array}\right.
$$

Studying this model, he found that intervention strategies decrease endemic levels and tend to make the dynamical behavior of a disease evolution simpler.

However, spatial diffusion is an assignable phenomenon. At present, lots of scholars have studied the epidemic models with spatial diffusion, and the traveling wave solutions play an important role in understanding the long-time asymptotic property of reactiondiffusion models [24-27]. In endemic systems, the existence of traveling wave solutions indicates possible transition from infectious individuals to susceptible individuals from the initial disease-free steady state to the endemic steady state. We consider some spatial aspects of the model in order to estimate the propagation speed and to describe the spation. Let $\Omega$ be a spatial habitat with smooth boundary $\partial \Omega$. We now incorporate the spatial content to the simplified model (1.2) and obtain the following PDE model for which each variable is a function of both the spatial location $x \in \mathbb{R}$ and the time $t \in \mathbb{R}$ :

$$
\left\{\begin{array}{l}
\frac{\partial I}{\partial t}=d_{I} \frac{\partial^{2} I}{\partial x^{2}}+\frac{a I}{f(I)}(N-I-R)-(d+\gamma) I \\
\frac{\partial R}{\partial t}=d_{R} \frac{\partial^{2} R}{\partial x^{2}}+\gamma I-(d+b) R \\
\frac{\partial I}{\partial n}=\frac{\partial R}{\partial n}=0, \quad t>0, x \in \partial \Omega, \\
I(0, x)=I_{0}(x) \geq 0, \quad R(0, x)=R_{0}(x) \geq 0, \quad \forall x \in \bar{\Omega},
\end{array}\right.
$$


where $d_{I}>0$ and $d_{R}>0$ are the diffusion coefficients of $I$ and $R$, respectively, $\frac{\partial}{\partial n}$ denotes the outward normal derivative on $\partial \Omega$; the Neumann boundary conditions imply that the populations always move in $\Omega$ but do not move across the boundary $\partial \Omega$.

Throughout the paper, we assume that the function $f(I)$ satisfies the following condition:

$\left(\mathrm{A}_{1}\right) f(I)$ is increasing for $I>0$, and $f(0)>0$.

Furthermore, we make the different assumption from Wang [19]:

$\left(\mathrm{A}_{2}\right)$ The infection force $\frac{I}{f(I)}$ satisfies $\left(\frac{I}{f(I)}\right)^{\prime}>0$ for $I>0$, which is reasonable as a lot of endemic diseases extend seriously along with the increase of infectious individuals.

In addition, we assume:

$\left(\mathrm{A}_{3}\right)$ There exists a constant $L>0$ such that $\left|f\left(I_{1}\right)-f\left(I_{2}\right)\right| \leq L\left|I_{1}-I_{2}\right|$ for all $I_{1}, I_{2} \in \bar{\Omega}$.

The paper is organized as follows. In Section 2, we study the existence of equilibria and show some well-posedness results for system (1.3). In Section 3, we present a qualitative analysis of the model (1.3). We show the local and global stability of the associated steady states. In Section 4, we prove the existence of traveling wave solutions of system (1.3) by the upper-lower solution technique when $1<R_{0}<1+\frac{b+d}{\gamma}$. Numerical simulations are given to illustrate the analytical results in Section 5. A brief discussion and a summary of the findings in the study are provided in the last section.

\section{The well-posedness of system (1.3)}

Throughout this paper, we use the usual notation for the standard ordering in $\mathbb{R}^{2}$ or $X$. That is, for any $\phi=\left(\phi_{1}, \phi_{2}\right)$ and $\omega=\left(\omega_{1}, \omega_{2}\right)$, we denote $\phi \leq \omega$ if $\phi_{i} \leq \omega_{i}$ and $\phi<\omega$ if $\phi_{i} \leq \omega_{i}$ but $\phi \neq \omega$. The cone $\mathbb{R}_{+}^{2}$ denotes the subset of $\mathbb{R}^{2}$ with vectors $x \geq 0, X=C\left(\bar{\Omega}, \mathbb{R}^{2}\right)$ is the Banach space with the supremum norm $\|\cdot\|_{X}$. Define $X^{+}=C\left(\bar{\Omega}, \mathbb{R}_{+}^{2}\right)$; then $\left(X, X^{+}\right)$ is a strongly ordered space.

Obviously, the trivial equilibrium point $E_{0}=(0,0)$ is a disease-free equilibrium of $(1.3)$. By a similar argument as in [28] it is easy to show that the basic reproduction number of system (1.3) is $R_{0}=\frac{N a}{f(0)(d+\gamma)}$.

\section{Lemma 2.1}

(1) If $R_{0}<1$, then system (1.3) has a disease-free equilibrium $E_{0}=(0,0)$.

(2) If $R_{0}>1$, then system (1.3) admits a unique endemic steady state $E^{*}=\left(I^{*}, R^{*}\right)$, where

$$
R^{*}=\frac{\gamma I^{*}}{d+b}
$$

and $I^{*}$ is the unique root of

$$
\frac{\gamma I}{d+b}=N-I-\frac{d+\gamma}{a} f(I) .
$$

By direct computations we can get

$$
R^{*}+I^{*}=N-\frac{d+\gamma}{a} f\left(I^{*}\right)<N-\frac{d+\gamma}{a} f(0)=\frac{(d+\gamma) f(0)}{a}\left(R_{0}-1\right),
$$

that is, $0<I^{*}<\frac{N(b+d)}{\gamma+b+d}:=M_{1}, 0<R^{*}<\frac{N \gamma}{\gamma+b+d}:=M_{2}$, and $M_{1}+M_{2}=N$. 
Define $Q_{M}=\left\{\varphi=\left(\varphi_{1}, \varphi_{2}\right) \in X^{+}: \varphi_{1}+\varphi_{2} \leq N, \forall x \in \bar{\Omega}\right\}$. For any $\varphi=\left(\varphi_{1}, \varphi_{2}\right) \in Q_{M}$ and $x \in \bar{\Omega}$, define $G=\left(G_{1}, G_{2}\right): Q_{M} \rightarrow X$ by

$$
\begin{aligned}
& G_{1}(\varphi)(x)=\frac{a \varphi_{1}}{f\left(\varphi_{1}\right)}\left(N-\varphi_{1}-\varphi_{2}\right)-(d+\gamma) \varphi_{1}, \\
& G_{2}(\varphi)(x)=\gamma \varphi_{1}-(d+b) \varphi_{2} .
\end{aligned}
$$

Since $f$ is Lipschitz continuous, $G$ is Lipschitz continuous in any bounded subset of $\Omega_{M}$, and system (1.3) can be written as an abstract differential equation as follows:

$$
\begin{aligned}
& \frac{\mathrm{d} u}{\mathrm{~d} t}=A u+G(u), \quad t>0, \\
& u_{0}=\varphi \in Q_{M},
\end{aligned}
$$

where $u=(I, R), A u:=\left(d_{I} \frac{\partial^{2} I}{\partial x^{2}}, d_{R} \frac{\partial^{2} R}{\partial x^{2}}\right)$, and $\varphi=\left(\varphi_{1}, \varphi_{2}\right)$.

Theorem 2.1 For any given initial data $\varphi=\left(\varphi_{1}, \varphi_{2}\right) \in Q_{M}$, system (1.3) has a unique nonnegative solution $u(t, x, \varphi)$ on $[0, \infty) \times \bar{\Omega}$ with $u(0, \cdot, \varphi)=\varphi$. Furthermore, $u(t, \cdot, \varphi) \in Q_{M}$ for $t \geq 0$, and $u(t, x, \varphi)$ for $(t, x) \in[0, \infty) \times \bar{\Omega}$ is a classical solution of system (1.3).

Proof For any $\varphi=\left(\varphi_{1}, \varphi_{2}\right) \in Q_{M}$ and $h \geq 0$, we can get

$$
\varphi(x)+h G(\varphi)(x)=\left(\begin{array}{c}
\varphi_{1}+\frac{a h \varphi_{1}}{f\left(\varphi_{1}\right)}\left(N-\varphi_{1}-\varphi_{2}\right)-h(d+\gamma) \varphi_{1} \\
\varphi_{2}+h \gamma \varphi_{1}-h(d+b) \varphi_{2}
\end{array}\right) .
$$

For $0 \leq h<\min \left\{\frac{1}{d+\gamma}, \frac{1}{b+d}\right\}$, it follows that

$$
\begin{aligned}
& \varphi_{1}+\frac{a h \varphi_{1}}{f\left(\varphi_{1}\right)}\left(N-\varphi_{1}-\varphi_{2}\right)-h(d+\gamma) \varphi_{1} \geq \varphi_{1}(1-h(d+\gamma)) \geq 0, \\
& \varphi_{2}+h \gamma \varphi_{1}-h(d+b) \varphi_{2} \geq \varphi_{2}(1-h(b+d)) \geq 0 .
\end{aligned}
$$

For sufficiently small $h>0$ and all $\left(\varphi_{1}, \varphi_{2}\right) \in Q_{M}$, we have

$$
\begin{aligned}
\varphi_{1} & +\frac{a h \varphi_{1}}{f\left(\varphi_{1}\right)}\left(N-\varphi_{1}-\varphi_{2}\right)-h(d+\gamma) \varphi_{1}+\varphi_{2}+h \gamma \varphi_{1}-h(d+b) \varphi_{2} \\
& =\left(\varphi_{1}+\varphi_{2}\right)+\frac{a h \varphi_{1}}{f\left(\varphi_{1}\right)}\left(N-\varphi_{1}-\varphi_{2}\right)-h d \varphi_{1}-h(b+d) \varphi_{2} \\
& \leq\left(\varphi_{1}+\varphi_{2}\right)+\frac{a h N}{f(0)}\left(N-\varphi_{1}-\varphi_{2}\right) \\
& =\frac{a h N^{2}}{f(0)}+\left(\varphi_{1}+\varphi_{2}\right)\left(1-\frac{a h N}{f(0)}\right) \\
& \leq N
\end{aligned}
$$

Thus, it follows that $\varphi(x)+h G(\varphi)(x) \in Q_{M}$. This implies that

$$
\lim _{h \rightarrow 0^{+}} \frac{1}{h} \operatorname{dist}\left(\varphi(x)+h F(\varphi)(x), \Omega_{M}\right)=0, \quad \forall \varphi \in Q_{M} .
$$


Therefore, the existence and uniqueness of $u(t, x, \varphi)$ on $[0, \infty) \times \bar{\Omega}$ follow from [29] (Corollary 4 with zero delay) with $u(0, \cdot, \varphi)=\varphi$ and $u(t, \cdot, \varphi) \in Q_{M}$ for $t \geq 0$.

\section{Qualitative analysis}

In this section, we establish conditions for the local and global stability of the equilibria of system (1.3) by judging the symbol of the characteristic roots of Jacobian matrix and constructing suitable Lyapunov functions.

\subsection{Local dynamics of equilibria}

In this subsection, we discuss the local stability of constant steady states $E_{0}$ and $E^{*}$ of system (1.3) by analyzing the corresponding characteristic equation.

Suppose that $0=\mu_{1}<\mu_{2}<\cdots$ are the eigenvalues of the operator $-\triangle$ on $\Omega$ with Neumann boundary conditions and that $E\left(\mu_{i}\right)$ is the eigenspace corresponding to $\mu_{i}$ in $C^{1}(\Omega)$. Denote $X=\left[C^{1}(\Omega)\right]^{2}$, let $\left\{\phi_{i j}: j=1,2, \ldots, \operatorname{dim} E\left(\mu_{i}\right)\right\}$ be an orthonormal basis of $E\left(\mu_{i}\right)$, and $X_{i j}=\left\{c \phi_{i j}: c \in \mathbb{R}^{2}\right\}$. Then

$$
X:=\bigotimes_{i=1}^{\infty} X_{i}, \quad X_{i}:=\bigotimes_{j=1}^{\operatorname{dim} E\left(\mu_{i}\right)} X_{i j}
$$

Let

$$
J=\left(\frac{a I}{f(I)}(N-I-R)-(d+\gamma) I, \gamma I-(b+d) R\right), \quad E=(I, R) .
$$

Then the Jacobian matrix is

$$
J_{E}=\left(\begin{array}{cc}
a\left(\frac{I}{f(I)}\right)^{\prime}(N-I-R)-\frac{a I}{f(I)}-(d+\gamma) & -\frac{a I}{f(I)} \\
\gamma & -(b+d)
\end{array}\right) .
$$

Let $D=\operatorname{diag}\left(d_{I}, d_{R}\right)$, and let $U=(I, R)$ be any feasible steady state of system (1.3). Then the linearization of system (1.3) at $U=(I, R)$ is of the form $D \triangle+J_{E}(U)$. For each $i \geq 1, X_{i}$ is invariant under the operator $D \triangle+J_{E}(U)$, and $\lambda$ is an eigenvalue of $D \triangle+J_{E}(U)$ if and only if it is an eigenvalue of the matrix $-\mu_{i} D+J_{E}(U)$ for some $i \geq 1$, in which case, there is an eigenvector in $X_{i}$. Hence, we have the following characteristic matrix of system (1.3):

$$
\begin{aligned}
& \operatorname{det}\left(\lambda I_{0}+\mu_{i} D-J_{E}(U)\right) \\
& \quad=\left|\begin{array}{cc}
\lambda+\mu_{i} d_{I}-a\left(\frac{I}{f(I)}\right)^{\prime}(N-I-R)+\frac{a I}{f(I)}+(d+\gamma) & \frac{a I}{f(I)} \\
-\gamma & \lambda+\mu_{i} d_{R}+b+d
\end{array}\right| .
\end{aligned}
$$

Theorem 3.1 If $R_{0}<1$, then the disease-free equilibrium $E_{0}=(0,0)$ of system (1.3) is locally asymptotically stable.

Proof Let $(I, R)=(0,0)$ in $(3.1)$. Then the Jacobian determinant is given by

$$
\begin{aligned}
& \operatorname{det}\left(\lambda I_{0}+\mu_{i} D-J_{E_{0}}\right) \\
& \quad=\left|\begin{array}{cc}
\lambda+\mu_{i} d_{I}-\frac{a N}{f(0)}+(d+\gamma) & 0 \\
-\gamma & \lambda+\mu_{i} d_{R}+b+d
\end{array}\right|
\end{aligned}
$$




$$
\begin{aligned}
& =\left(\lambda+\mu_{i} d_{I}-\frac{a N}{f(0)}+(d+\gamma)\right)\left(\lambda+\mu_{i} d_{R}+b+d\right) \\
& =0 .
\end{aligned}
$$

Therefore, for any $\mu_{i} \geq 0$, (3.2) has a real root $\lambda_{1}=-\mu_{i} d_{R}-b-d<0$, and when $R_{0}<1$, the other root $\lambda_{2}=-\mu_{i} d_{I}+\frac{a N}{f(0)}-(d+\gamma)<0$. Thus, both roots of (3.2) have negative real parts. Hence, $E_{0}(0,0)$ is locally asymptotically stable when $R_{0}<1$.

Theorem 3.2 If $R_{0}>1$, then the endemic equilibrium $E^{*}=\left(I^{*}, R^{*}\right)$ of system (1.3) is locally asymptotically stable.

Proof Let $(I, R)=\left(I^{*}, R^{*}\right)$. Then the Jacobian determinant is given by

$$
\begin{aligned}
& \operatorname{det}\left(\lambda I_{0}+\mu_{i} D-J_{\left.E^{*}\right)}\right. \\
& =\left|\begin{array}{cc}
\lambda+\mu_{i} d_{I}-\frac{a\left(N-2 I^{*}-R^{*}\right)}{f\left(I^{*}\right)}+d+\gamma+\frac{a\left(N-I^{*}-R^{*}\right) I^{*} f^{\prime}\left(I^{*}\right)}{f^{2}\left(I^{*}\right)} & \frac{a I^{*}}{f\left(I^{*}\right)} \\
-\gamma & \lambda+\mu_{i} d_{R}+b+d
\end{array}\right| \\
& =\left|\begin{array}{cc}
\lambda+\mu_{i} d_{I}-\frac{(d+\gamma)\left(N-2 I^{*}-R^{*}\right)}{N-I^{*}-R^{*}}+d+\gamma+\frac{I^{*} f^{\prime}\left(I^{*}\right)(d+\gamma)^{2}}{a\left(N-I^{*}-R^{*}\right)} & \frac{I^{*}(d+\gamma)}{N-I^{*}-R^{*}} \\
-\gamma & \lambda+\mu_{i} d_{R}+b+d
\end{array}\right| \\
& =\lambda^{2}+A_{1} \lambda+A_{2},
\end{aligned}
$$

where

$$
\begin{aligned}
A_{1}= & \mu_{i}\left(d_{I}+d_{R}\right)+\frac{b\left(N-I^{*}-R^{*}\right)+d\left(N-R^{*}\right)+\gamma I^{*}}{N-I^{*}-R^{*}}+\frac{I^{*} f^{\prime}\left(I^{*}\right)(d+\gamma)^{2}}{a\left(N-I^{*}-R^{*}\right)}>0, \\
A_{2}= & {\left[\mu_{i} d_{I}-\frac{(d+\gamma)\left(N-2 I^{*}-R^{*}\right)}{N-I^{*}-R^{*}}+d+\gamma\right.} \\
& \left.+\frac{I^{*} f^{\prime}\left(I^{*}\right)(d+\gamma)^{2}}{a\left(N-I^{*}-R^{*}\right)}\right]\left(\mu_{i} d_{R}+b+d\right)+\frac{\gamma I^{*}(d+\gamma)}{N-I^{*}-R^{*}} .
\end{aligned}
$$

Clearly, $d+\gamma-\frac{(d+\gamma)\left(N-2 I^{*}-R^{*}\right)}{N-I^{*}-R^{*}}=\frac{(d+\gamma) I^{*}}{N-I^{*}-R^{*}}>0$, so $A_{2}>0$. By Routh-Hurwitz criterion, both roots of (3.3) have negative real parts. Therefore, the endemic equilibrium $E^{*}\left(I^{*}, R^{*}\right)$ is locally asymptotically stable when $R_{0}>1$.

\subsection{Global stability}

In this subsection, we study the global stability of the endemic equilibrium $E^{*}$ and the disease-free equilibrium $E_{0}$ of system (1.3). The technique of the proofs is based on construction of appropriate Lyapunov functions. Anything else, the techniques of couple lowupper solutions, monotone iteration, and so on are frequently used to work on the global stability of the constant steady states of the diffusive model; see [27, 30, 31].

We have the following result.

Theorem 3.3 If $R_{0} \leq 1$, then the disease-free steady state $E_{0}=(0,0)$ of system (1.3) is globally asymptotically stable.

Proof Define the Lyapunov function

$$
V_{1}(t)=\int_{\Omega} W_{1}(x, t) \mathrm{d} x=\int_{\Omega} I+\frac{(d+\gamma)\left(1-R_{0}\right)}{\gamma} R \mathrm{~d} x .
$$


Then

$$
\begin{aligned}
\frac{\partial V_{1}}{\partial t}= & \int_{\Omega} \frac{\partial W_{1}}{\partial t} \mathrm{~d} x=\int_{\Omega}\left[\frac{\partial I}{\partial t}+\frac{(d+\gamma)\left(1-R_{0}\right)}{\gamma} \frac{\partial R}{\partial t}\right] \mathrm{d} x \\
= & \int_{\Omega}\left[d_{I} \Delta I+\frac{(d+\gamma)\left(1-R_{0}\right)}{\gamma} d_{R} \Delta R+\frac{a N I}{f(I)}-\frac{a I^{2}}{f(I)}-\frac{a I R}{f(I)}\right. \\
& \left.-(d+\gamma) I+(d+\gamma)\left(1-R_{0}\right) I-\frac{(d+\gamma)\left(1-R_{0}\right)(b+d)}{\gamma} R\right] \mathrm{d} x .
\end{aligned}
$$

Clearly,

$$
\begin{aligned}
& \frac{a N I}{f(I)}-(d+\gamma) I+(d+\gamma)\left(1-R_{0}\right) I \\
& \quad \leq\left[\frac{a N}{f(0)}-(d+\gamma) R_{0}\right] I \\
& \quad=(d+\gamma)\left[\frac{a N}{f(0)(d+\gamma)}-R_{0}\right] I \\
& \quad=0 .
\end{aligned}
$$

By the Neumann boundary conditions it is easy to show that

$$
\int_{\Omega} \Delta I \mathrm{~d} x=\int_{\Omega} \triangle R \mathrm{~d} x=0
$$

Hence, for any $t>0$ and all $I, R \geq 0, \frac{\partial V_{1}}{\partial t} \leq 0$ as $R_{0} \leq 1$. Obviously, $\frac{\partial V_{1}}{\partial t}=0$ if and only if $I=0$ and $R=0$. It is easy to get that the singleton $E_{0}$ is the largest compact invariant set in $\left\{(I, R) \in Q_{M}: \frac{\partial V_{1}}{\partial t}=0\right\}$. Because the forward orbit of system (1.3) is compact, $\lim _{t \rightarrow \infty}(I(\cdot, t), R(\cdot, t))=E_{0}$ by the LaSalle invariance principle [32], Theorem 4.3.4. Thus, $E_{0}=(0,0)$ is globally asymptotically stable when $R_{0} \leq 1$.

Lemma 3.1 Let $R_{0}>1$. Define the function

$$
W(I)=f\left(I^{*}\right)\left(I-I^{*}-I^{*} \ln \frac{I}{I^{*}}\right)+\int_{I^{*}}^{I} \frac{\left(f(u)-f\left(I^{*}\right)\right)\left(u-I^{*}\right)}{u} \mathrm{~d} u .
$$

Then $W(I) \geq 0$. Furthermore, the equality holds if and only if $I=I^{*}$.

Proof By immediate calculation,

$$
\frac{\mathrm{d} W}{\mathrm{~d} I}=\frac{f(I)}{I}\left(I-I^{*}\right)
$$

Since $f(I)>0$ and $I>0$, we have $\frac{\mathrm{d} W}{\mathrm{~d} I}=0$ if $I=I^{*}$, that is, $I=I^{*}$ is a unique stationary point of $W$. We have $\frac{\mathrm{d} W}{\mathrm{~d} l}>0$ for $I>I^{*}$ and $\frac{\mathrm{d} W}{\mathrm{~d} t}<0$ for $I<I^{*}$, that is, $I^{*}$ is the unique minimum point of $W$. Further, $W\left(I^{*}\right)=0$, so that $W(I) \geq W\left(I^{*}\right)=0$.

Theorem 3.4 If $R_{0}>1$, then the endemic equilibrium $E^{*}=\left(I^{*}, R^{*}\right)$ of system $(1.3)$ is globally asymptotically stable. 
Proof Define the Lyapunov function

$$
V=\int_{\Omega} W+\frac{1}{2} k\left(R-R^{*}\right)^{2} \mathrm{~d} x
$$

Then

$$
\begin{aligned}
\frac{\partial V}{\partial t}= & \int_{\Omega} \frac{\mathrm{d} W}{\mathrm{~d} I} \frac{\partial I}{\partial t}+k\left(R-R^{*}\right) \frac{\partial R}{\partial t} \mathrm{~d} x \\
= & \int_{\Omega} d_{I} f(I) \frac{I-I^{*}}{I} \Delta I \mathrm{~d} x+\int_{\Omega} k d_{R}\left(R-R^{*}\right) \Delta R \mathrm{~d} x \\
& +\int_{\Omega} \frac{f(I)}{I}\left(I-I^{*}\right)\left[\frac{a I}{f(I)}\left(-\left(I-I^{*}\right)-\left(R-R^{*}\right)+\frac{d+\gamma}{a}\left(f\left(I^{*}\right)-f(I)\right)\right)\right] \\
& +k\left(R-R^{*}\right)\left(\gamma\left(I-I^{*}\right)-(b+d)\left(R-R^{*}\right)\right) \mathrm{d} x \\
= & -\int_{I} d_{I}\|\nabla I\|^{2} f^{\prime}(I) \mathrm{d} x-\int_{\Omega} d_{I} I^{*}\left(\frac{f(I)}{I}\right)^{2}\left(\frac{I}{f(I)}\right)^{\prime}\|\nabla I\|^{2} \mathrm{~d} x-\int_{\Omega} k d_{R}\|\nabla R\|^{2} \mathrm{~d} x \\
& -\int_{\Omega} a\left(I-I^{*}\right)^{2}+k(b+d)\left(R-R^{*}\right)^{2}+(d+\gamma)\left(I-I^{*}\right)\left(f(I)-f\left(I^{*}\right)\right) \mathrm{d} x \\
& +\int_{\Omega}(k \gamma-a)\left(R-R^{*}\right)\left(I-I^{*}\right) \mathrm{d} x .
\end{aligned}
$$

Let $k=\frac{a}{\gamma}$. Then $\frac{\partial V}{\partial t} \leq 0$ for all $t>0, I>0, R>0$, and $\frac{\partial V}{\partial t}=0$ iff $(I, R)=\left(I^{*}, R^{*}\right)$. Obviously, $E^{*}$ is the largest compact invariant set in $\left\{(I, R) \in Q_{M}: \frac{\partial V_{1}}{\partial t}=0\right\}$. Since the forward orbit of system (1.3) is compact, $\lim _{t \rightarrow \infty}(I(\cdot, t), R(\cdot, t))=E^{*}$ by the LaSalle invariance principle [32], Theorem 4.3.4. Thus, the equilibrium $E^{*}=\left(I^{*}, R^{*}\right)$ is globally asymptotically stable if $R_{0}>1$.

\section{Traveling wave solution}

A traveling wave solution of system (1.3) is a special translation-invariant solution of the form $I(x, t)=\phi_{1}(\xi), R(x, t)=\phi_{2}(\xi), \xi=x+c t$, where $c>0$ is the wave speed, and $\phi_{1}, \phi_{2}$ are the profiles of the wave that propagates through the one-dimensional spatial domain. For simplicity, denote $d_{I}, d_{R}, \xi$ as $d_{1}, d_{2}, t$, respectively. Substituting this special solution into system (1.3), we obtain the corresponding wave equation

$$
\begin{aligned}
& d_{1} \phi_{1}^{\prime \prime}(t)-c \phi_{1}^{\prime}(t)+\frac{a \phi_{1}(t)}{f\left(\phi_{1}(t)\right)}\left(N-\phi_{1}(t)-\phi_{2}(t)\right)-(d+\gamma) \phi_{1}(t)=0, \\
& d_{2} \phi_{2}^{\prime \prime}(t)-c \phi_{2}^{\prime}(t)+\gamma \phi_{1}(t)-(b+d) \phi_{2}(t)=0 .
\end{aligned}
$$

Consider the nontrivial solution $\left(\phi_{1}(t), \phi_{2}(t)\right)$ to system (4.1) with the following boundary conditions:

$$
\lim _{t \rightarrow-\infty}\left(\phi_{1}(t), \phi_{2}(t)\right)=(0,0), \quad \lim _{t \rightarrow+\infty}\left(\phi_{1}(t), \phi_{2}(t)\right)=\left(I^{*}, R^{*}\right)
$$

where $\left(I^{*}, R^{*}\right)$ are defined in Lemma 2.1. For convenience, set

$$
\begin{aligned}
& h_{1}\left(\phi_{1}, \phi_{2}\right)(t)=\frac{a \phi_{1}(t)}{f\left(\phi_{1}(t)\right)}\left(N-\phi_{1}(t)-\phi_{2}(t)\right)-(d+\gamma) \phi_{1}(t), \\
& h_{2}\left(\phi_{1}, \phi_{2}\right)(t)=\gamma \phi_{1}(t)-(b+d) \phi_{2}(t) .
\end{aligned}
$$


Then system (4.1) can be rewritten as

$$
\left\{\begin{array}{l}
d_{1} \phi_{1}^{\prime \prime}(t)-c \phi_{1}^{\prime}(t)+h_{1}\left(\phi_{1}, \phi_{2}\right)(t)=0 \\
d_{2} \phi_{2}^{\prime \prime}(t)-c \phi_{2}^{\prime}(t)+h_{2}\left(\phi_{1}, \phi_{2}\right)(t)=0
\end{array}\right.
$$

Definition 4.1 A pair of functions $\bar{\phi}=\left(\bar{\phi}_{1}, \bar{\phi}_{2}\right)$ and $\underline{\phi}=\left(\underline{\phi}_{1}, \underline{\phi}_{2}\right) \in C\left(\mathbb{R}, \mathbb{R}^{2}\right)$ are called a couple of upper and lower solutions of system (4.1), respectively, if there exists a finite set $\Sigma=\left\{t_{1}, t_{2}, \ldots, t_{m}\right\}$ such that $\bar{\phi}^{\prime \prime}(t), \bar{\phi}^{\prime}(t), \underline{\phi}^{\prime \prime}(t), \underline{\phi^{\prime}}(t)$ are continuous on $\mathbb{R} \backslash \Sigma$ and satisfy

$$
\begin{aligned}
& \left\{\begin{array}{l}
d_{1} \bar{\phi}_{1}^{\prime \prime}(t)-c \bar{\phi}_{1}^{\prime}(t)+\frac{a \bar{\phi}_{1}(t)}{f\left(\bar{\phi}_{1}(t)\right)}\left(N-\bar{\phi}_{1}(t)-\underline{\phi}_{2}(t)\right)-(d+\gamma) \bar{\phi}_{1}(t) \leq 0, \\
d_{2} \bar{\phi}_{2}^{\prime \prime}(t)-c \bar{\phi}_{2}^{\prime}(t)+\gamma \bar{\phi}_{1}(t)-(b+d) \bar{\phi}_{2}(t) \leq 0,
\end{array}\right. \\
& \left\{\begin{array}{l}
d_{1} \underline{\phi}_{1}^{\prime \prime}(t)-c \underline{\phi}_{1}^{\prime}(t)+\frac{a \phi_{1}(t)}{f\left(\underline{\phi}_{1}(t)\right)}\left(N-\underline{\phi}_{1}(t)-\bar{\phi}_{2}(t)\right)-(d+\gamma) \underline{\phi}_{1}(t) \geq 0, \\
d_{2} \underline{\phi}_{2}^{\prime \prime}(t)-c \underline{\phi}_{2}^{\prime}(t)+\gamma \underline{\phi}_{1}(t)-(b+d) \underline{\phi}_{2}(t) \geq 0 .
\end{array}\right.
\end{aligned}
$$

The functions $\bar{\phi}, \underline{\phi}$ also satisfy the following conditions:

$\left(\mathrm{B}_{1}\right) \quad 0 \leq\left(\underline{\phi}_{1}, \underline{\phi}_{2}\right) \leq\left(\bar{\phi}_{1}, \bar{\phi}_{2}\right) \leq M=\left(M_{1}, M_{2}\right)$;

$\left(\mathrm{B}_{2}\right) \lim _{t \rightarrow-\infty}\left(\bar{\phi}_{1}(t), \bar{\phi}_{2}(t)\right)=(0,0), \lim _{t \rightarrow \infty}\left(\underline{\phi}_{1}(t), \underline{\phi}_{2}(t)\right)=\lim _{t \rightarrow \infty}\left(\bar{\phi}_{1}(t), \bar{\phi}_{2}(t)\right)=\left(I^{*}, R^{*}\right)$;

$\left(\mathrm{B}_{3}\right) \bar{\phi}_{i}^{\prime}\left(t^{+}\right) \leq \bar{\phi}_{i}^{\prime}\left(t^{-}\right), \underline{\phi}_{i}^{\prime}\left(t^{-}\right) \leq \underline{\phi}_{i}^{\prime}\left(t^{+}\right), t \in R, i=1,2$.

Let $D=(d+\gamma) R_{0}$ and $d_{m}=\max \left\{d_{1}, d_{2}\right\}$. When $c>c^{*}=2 \sqrt{d_{m} D}$, the equation $d_{m} \lambda^{2}-c \lambda+$ $D=0$ has two roots

$$
\lambda_{1}=\frac{c-\sqrt{c^{2}-4 d_{m} D}}{2 d_{m}}, \quad \lambda_{2}=\frac{c+\sqrt{c^{2}-4 d_{m} D}}{2 d_{m}}
$$

and there exists $\lambda_{0}$ such that $d_{m} \lambda_{0}^{2}-c \lambda_{0}+D \leq 0$ when $\lambda_{1}<\lambda_{0}<\lambda_{2}$.

We can choose appropriate $\varepsilon_{i}>0(i=1, \ldots, 4)$, satisfying the following conditions:

$$
\begin{aligned}
& \varepsilon_{4}<\varepsilon_{1}, \quad \quad \varepsilon_{2}<\varepsilon_{3}<I^{*}, \\
& \gamma \varepsilon_{1}<\varepsilon_{2}(b+d), \quad \gamma \varepsilon_{3}<\varepsilon_{4}(b+d),
\end{aligned}
$$

and $\varepsilon_{1}$ and $\varepsilon_{2}$ also satisfy the following inequalities

$$
\frac{M_{1}}{I^{*}}<\frac{\varepsilon_{1}}{M_{1}-I^{*}}, \quad \frac{M_{2}}{R^{*}}<\frac{\varepsilon_{2}}{M_{2}-R^{*}} .
$$

Next, we construct a pair of upper and lower solutions for system (1.3). In consideration of the above constant $\varepsilon_{i}$ satisfying (4.6) and (4.7), we now define two continuous functions $\bar{\phi}(t)=\left(\bar{\phi}_{1}(t), \bar{\phi}_{2}(t)\right)$ and $\underline{\phi}(t)=\left(\underline{\phi}_{1}(t), \underline{\phi}_{2}(t)\right)$ for all $t \in \mathbb{R}$ as follows:

$$
\begin{aligned}
& \bar{\phi}_{1}=\left\{\begin{array}{ll}
I^{*} e^{\lambda_{0} t}, & t \leq t_{1}, \\
M_{1}, & t_{1}<t \leq t_{2}, \\
I^{*}+\varepsilon_{1} e^{-\lambda t}, & t>t_{2},
\end{array} \quad \bar{\phi}_{2}= \begin{cases}R^{*} e^{\lambda_{0} t}, & t \leq t_{3}, \\
M_{2}, & t_{3}<t \leq t_{4}, \\
R^{*}+\varepsilon_{2} e^{-\lambda t} & t>t_{4},\end{cases} \right. \\
& \underline{\phi}_{1}=\left\{\begin{array}{ll}
0, & t \leq t_{5}, \\
I^{*}-\varepsilon_{3} e^{-\lambda t}, & t>t_{5},
\end{array} \quad \underline{\phi}_{2}= \begin{cases}0, & t \leq t_{6}, \\
R^{*}-\varepsilon_{4} e^{-\lambda t}, & t>t_{6},\end{cases} \right.
\end{aligned}
$$


where

$$
\begin{aligned}
& t_{1}=t_{3}=\frac{1}{\lambda_{0}} \ln \frac{M_{1}}{I^{*}}=\frac{1}{\lambda_{0}} \ln \frac{M_{2}}{R^{*}}, \\
& t_{2}=\frac{1}{\lambda} \ln \frac{\varepsilon_{1}}{M_{1}-I^{*}}, \quad t_{4}=\frac{1}{\lambda} \ln \frac{\varepsilon_{2}}{M_{2}-R^{*}}, \\
& t_{5}=\frac{1}{\lambda} \ln \frac{\varepsilon_{3}}{I^{*}}, \quad t_{6}=\frac{1}{\lambda} \ln \frac{\varepsilon_{4}}{R^{*}},
\end{aligned}
$$

and $\lambda \in\left(0, \lambda_{0}\right)$ is a constant. It is obvious that $t_{1}<t_{2}, t_{3}<t_{4}$ by (4.7). Clearly, $\bar{\phi}_{1}, \bar{\phi}_{2}, \underline{\phi}_{1}$, $\underline{\phi}_{2}$ satisfy $\left(\mathrm{B}_{1}\right),\left(\mathrm{B}_{2}\right),\left(\mathrm{B}_{3}\right)$. Next, we prove that $\bar{\phi}(t), \underline{\phi}(t)$ are upper and lower solutions of system (4.1) under some suitable conditions.

Lemma 4.1 If $1<R_{0}<1+\frac{b+d}{\gamma}$, then $\bar{\phi}=\left(\bar{\phi}_{1}, \bar{\phi}_{2}\right)$ is an upper solution of system (4.1).

Proof (1) For $\bar{\phi}_{1}(t)$,

$$
N_{1}\left(\bar{\phi}_{1}\right)=d_{1} \bar{\phi}_{1}^{\prime \prime}(t)-c \bar{\phi}_{1}^{\prime}(t)+\frac{a \bar{\phi}_{1}(t)}{f\left(\bar{\phi}_{1}(t)\right)}\left(N-\bar{\phi}_{1}(t)-\underline{\phi}_{2}(t)\right)-(d+\gamma) \bar{\phi}_{1}(t) .
$$

Case 1. When $t \leq t_{1}, \bar{\phi}_{1}(t)=I^{*} e^{\lambda_{0} t}$, and

$$
\begin{aligned}
N_{1}\left(\bar{\phi}_{1}\right) & =I^{*} e^{\lambda_{0} t}\left(d_{1} \lambda_{0}^{2}-c \lambda_{0}+\frac{a\left(N-I^{*} e^{\lambda_{0} t}-\underline{\phi}_{2}(t)\right)}{f\left(I^{*} e^{\lambda_{0} t}\right)}-(d+\gamma)\right) \\
& \leq I^{*} e^{\lambda_{0} t}\left(d_{1} \lambda_{0}^{2}-c \lambda_{0}+(d+\gamma)\left(R_{0}-1\right)\right) \\
& \leq I^{*} e^{\lambda_{0} t}\left(d_{m} \lambda_{0}^{2}-c \lambda_{0}+D\right)=0 .
\end{aligned}
$$

Case 2. When $t_{1}<t \leq t_{2}, \bar{\phi}_{1}(t)=M_{1}$, and

$$
\begin{aligned}
N_{1}\left(\bar{\phi}_{1}\right) & =0-0+\frac{a M_{1}}{f\left(M_{1}\right)}\left(N-M_{1}-\underline{\phi}_{2}(t)\right)-(d+\gamma) M_{1} \\
& \leq M_{1}\left(\frac{a M_{2}}{f(0)}-(d+\gamma)\right) \\
& =M_{1}(d+\gamma)\left(\frac{R_{0} \gamma}{\gamma+b+d}-1\right) \\
& \leq 0 .
\end{aligned}
$$

Case 3. When $t>t_{2}, \bar{\phi}_{1}(t)=I^{*}+\varepsilon_{1} e^{-\lambda t}$, and

$$
\begin{aligned}
N_{1}\left(\bar{\phi}_{1}\right)= & d_{1} \varepsilon_{1} \lambda^{2} e^{-\lambda t}+c \varepsilon_{1} \lambda e^{-\lambda t}-(d+\gamma)\left(I^{*}+\varepsilon_{1} e^{-\lambda t}\right) \\
& +\frac{a\left(I^{*}+\varepsilon_{1} e^{-\lambda t}\right)}{f\left(I^{*}+\varepsilon_{1} e^{-\lambda t}\right)}\left(N-I^{*}-\varepsilon_{1} e^{-\lambda t}-R^{*}+\varepsilon_{4} e^{-\lambda t}\right) \\
\leq & \varepsilon_{1} d_{1} \lambda^{2} e^{-\lambda t}+c \varepsilon_{1} \lambda e^{-\lambda t}-(d+\gamma)\left(I^{*}+\varepsilon_{1} e^{-\lambda t}\right) \\
& +\frac{a\left(I^{*}+\varepsilon_{1} e^{-\lambda t}\right)}{f\left(I^{*}\right)}\left(\frac{d+\gamma}{a} f\left(I^{*}\right)-\left(\varepsilon_{1}-\varepsilon_{4}\right) e^{-\lambda t}\right) \\
\leq & e^{-\lambda t}\left(\varepsilon_{1} d_{1} \lambda^{2}+c \varepsilon_{1} \lambda-\frac{a\left(I^{*}\right)}{f\left(I^{*}\right)}\left(\varepsilon_{1}-\varepsilon_{4}\right)\right) .
\end{aligned}
$$


Defining

$$
L_{1}(\lambda)=\varepsilon_{1} d_{1} \lambda^{2}+c \varepsilon_{1} \lambda-\frac{a\left(I^{*}\right)}{f\left(I^{*}\right)}\left(\varepsilon_{1}-\varepsilon_{4}\right)
$$

by (4.6) we have $L_{1}(0)=-\frac{a\left(I^{*}\right)}{f\left(I^{*}\right)}\left(\varepsilon_{1}-\varepsilon_{4}\right)<0$. Thus, there exists $\lambda_{1}^{*}>0$ such that $L_{1}(\lambda) \leq 0$ for all $\lambda \in\left(0, \lambda_{1}^{*}\right)$, that is, $N_{1}\left(\bar{\phi}_{1}\right) \leq 0$ for all $\lambda \in\left(0, \lambda_{1}^{*}\right)$.

(2) For $\bar{\phi}_{2}(t)$,

$$
N_{2}\left(\bar{\phi}_{2}\right)=d_{2} \bar{\phi}_{2}^{\prime \prime}(t)-c \bar{\phi}_{2}^{\prime}(t)+\gamma \bar{\phi}_{1}(t)-(b+d) \bar{\phi}_{2}(t)
$$

Case 4. When $t \leq t_{3}, \bar{\phi}_{2}=R^{*} e^{\lambda_{0} t}$, and

$$
N_{2}\left(\bar{\phi}_{2}\right)=R^{*} e^{\lambda_{0} t}\left(d_{2} \lambda_{0}^{2}-c \lambda_{0}\right) \leq R^{*} e^{\lambda_{0} t}\left(d_{m} \lambda_{0}^{2}-c \lambda_{0}+D\right) \leq 0 .
$$

Case 5. When $t_{3}<t \leq t_{4}, \bar{\phi}_{2}(t)=M_{2}$, and

$$
N_{2}\left(\bar{\phi}_{2}\right)=0-0+\gamma \bar{\phi}_{1}(t)-(b+d) M_{2} \leq \gamma M_{1}-(b+d) M_{2}=0 .
$$

Case 6. When $t>t_{4}, \bar{\phi}_{2}=R^{*}+\varepsilon_{2} e^{-\lambda t}$, and

$$
\begin{aligned}
N_{2}\left(\bar{\phi}_{2}\right) & =\varepsilon_{2} d_{2} \lambda^{2} e^{-\lambda t}+c \varepsilon_{2} \lambda e^{-\lambda t}+\gamma\left(I^{*}+\varepsilon_{1} e^{-\lambda t}\right)-(b+d)\left(R^{*}+\varepsilon_{2} e^{-\lambda t}\right) \\
& =e^{-\lambda t}\left(\varepsilon_{2} d_{2} \lambda^{2}+c \varepsilon_{2} \lambda+\gamma \varepsilon_{1}-(b+d) \varepsilon_{2}\right) .
\end{aligned}
$$

Defining

$$
L_{2}(\lambda)=\varepsilon_{2} d_{2} \lambda^{2}+c \varepsilon_{2} \lambda+\gamma \varepsilon_{1}-(b+d) \varepsilon_{2},
$$

by the third inequality of (4.6) we have $L_{2}(0)=\gamma \varepsilon_{1}-(b+d) \varepsilon_{2}<0$. Thus, there exists $\lambda_{2}^{*}>0$ such that $L_{2}(\lambda) \leq 0$ for all $\lambda \in\left(0, \lambda_{2}^{*}\right)$, that is, $N_{2}\left(\bar{\phi}_{2}\right) \leq 0$ for all $\lambda \in\left(0, \lambda_{2}^{*}\right)$. Consequently, choosing $0<\lambda<\min \left\{\lambda_{1}^{*}, \lambda_{2}^{*}\right\}$, we get that $\bar{\phi}$ is an upper solution of system (1.3).

Lemma 4.2 If $R_{0}>1$, then $\underline{\phi}=\left(\underline{\phi}_{1}, \underline{\phi}_{2}\right)$ is a lower solution of system (4.1).

Proof (1) For $\underline{\phi}_{1}(t)$,

$$
N_{3}\left(\underline{\phi}_{1}\right)=d_{1} \underline{\phi}_{1}^{\prime \prime}(t)-c \underline{\phi}_{1}^{\prime}(t)+\frac{a \underline{\phi}_{1}(t)}{f\left(\underline{\phi}_{1}(t)\right)}\left(N-\underline{\phi}_{1}(t)-\bar{\phi}_{2}(t)\right)-(d+\gamma) \underline{\phi}_{1}(t)
$$

Case 1. When $t \leq t_{5}, \underline{\phi}_{1}(t)=0$, and $N_{3}\left(\underline{\phi}_{1}\right)=0 \geq 0$.

Case 2. When $t>t_{5}, \underline{\phi}_{1}(t)=I^{*}-\varepsilon_{3} e^{-\lambda t}$, and

$$
\begin{aligned}
N_{3}\left(\underline{\phi}_{1}\right)= & -\varepsilon_{3} d_{1} \lambda^{2} e^{-\lambda t}-c \varepsilon_{3} \lambda e^{-\lambda t}-(d+\gamma)\left(I^{*}-\varepsilon_{3} e^{-\lambda t}\right) \\
& +\frac{a\left(I^{*}-\varepsilon_{3} e^{-\lambda t}\right)}{f\left(I^{*}-\varepsilon_{3} e^{-\lambda t}\right)}\left(N-I^{*}+\varepsilon_{3} e^{-\lambda t}-R^{*}-\varepsilon_{2} e^{-\lambda t}\right) \\
\geq & -\varepsilon_{3} d_{1} \lambda^{2} e^{-\lambda t}-c \varepsilon_{3} \lambda e^{-\lambda t}-(d+\gamma)\left(I^{*}-\varepsilon_{3} e^{-\lambda t}\right)
\end{aligned}
$$




$$
\begin{aligned}
& +\frac{a\left(I^{*}-\varepsilon_{3} e^{-\lambda t}\right)}{f\left(I^{*}\right)}\left(\frac{d+\gamma}{a} f\left(I^{*}\right)+\left(\varepsilon_{3}-\varepsilon_{2}\right) e^{-\lambda t}\right) \\
\geq & e^{-\lambda t}\left(-\varepsilon_{3} d_{1} \lambda^{2}-c \varepsilon_{3} \lambda+\frac{a\left(I^{*}-\varepsilon_{3} e^{-\lambda\left(t-t_{5}\right) e^{-\lambda t_{5}}}\right)}{f\left(I^{*}\right)}\left(\varepsilon_{3}-\varepsilon_{2}\right)\right) \\
\geq & e^{-\lambda t}\left(-\varepsilon_{3} d_{1} \lambda^{2}-c \varepsilon_{3} \lambda+\frac{a\left(I^{*}-\varepsilon_{3} e^{-\lambda t_{5}}\right)}{f\left(I^{*}\right)}\left(\varepsilon_{3}-\varepsilon_{2}\right)\right) .
\end{aligned}
$$

Defining

$$
L_{3}(\lambda)=-\varepsilon_{3} d_{1} \lambda^{2}-c \varepsilon_{3} \lambda+\frac{a\left(I^{*}-\varepsilon_{3} e^{-\lambda t_{5}}\right)}{f\left(I^{*}\right)}\left(\varepsilon_{3}-\varepsilon_{2}\right)
$$

by (4.6) we have $L_{3}(0)=\frac{a\left(I^{*}-\varepsilon_{3}\right)}{f\left(I^{*}\right)}\left(\varepsilon_{3}-\varepsilon_{2}\right)>0$. Thus, by the continuity of $L_{3}(\lambda)$ in $\lambda$, there exists $\lambda_{3}^{*}>0$ such that $L_{3}(\lambda) \geq 0$ for all $\lambda \in\left(0, \lambda_{3}^{*}\right)$, that is, $N_{3}\left(\underline{\phi}_{1}\right) \geq 0$ for all $\lambda \in\left(0, \lambda_{3}^{*}\right)$.

(2) For $\underline{\phi}_{2}(t)$,

$$
N_{4}\left(\underline{\phi}_{2}\right)=d_{2} \underline{\phi}_{2}^{\prime \prime}(t)-c \underline{\phi}_{2}^{\prime}(t)+\gamma \underline{\phi}_{1}-(d+b) \underline{\phi}_{2}
$$

Case 3. When $t \leq t_{6}, \underline{\phi}_{2}(t)=0$, and $N_{4}\left(\underline{\phi}_{2}\right)=0-0+\gamma \underline{\phi}_{1} \geq 0$.

Case 4. When $t>t_{6}, \underline{\phi}_{2}(t)=R^{*}-\varepsilon_{4} e^{-\lambda t}$, and

$$
\begin{aligned}
N_{4}\left(\underline{\phi}_{2}\right) & =-\varepsilon_{4} d_{2} \lambda^{2} e^{-\lambda t}-c \varepsilon_{4} \lambda e^{-\lambda t}+\gamma\left(I^{*}-\varepsilon_{3} e^{-\lambda t}\right)-(b+d)\left(R^{*}-\varepsilon_{4} e^{-\lambda t}\right) \\
& =e^{-\lambda t}\left(-\varepsilon_{4} d_{2} \lambda^{2}-c \varepsilon_{4} \lambda-\gamma \varepsilon_{3}+\varepsilon_{4}(b+d)\right) .
\end{aligned}
$$

Defining

$$
L_{4}(\lambda)=-\varepsilon_{4} d_{2} \lambda^{2}-c \varepsilon_{4} \lambda-\gamma \varepsilon_{3}+\varepsilon_{4}(b+d)
$$

we have $L_{4}(0)=-\gamma \varepsilon_{3}+\varepsilon_{4}(b+d)>0$. Thus, there exists $\lambda_{4}^{*}>0$ such that $L_{4}(\lambda) \geq 0$ for all $\lambda \in$ $\left(0, \lambda_{4}^{*}\right)$, that is, $N_{4}\left(\underline{\phi}_{2}\right) \geq 0$ for all $\lambda \in\left(0, \lambda_{4}^{*}\right)$. Consequently, choosing $0<\lambda<\min \left\{\lambda_{3}^{*}, \lambda_{4}^{*}\right\}$, we get that $\underline{\phi}$ is an upper solution of system (1.3).

Next, we give our main results for the existence of traveling wave solutions for system (1.3).

Choose two positive constants $\beta_{1} \geq d+\gamma+\frac{a N L M_{1}}{f^{2}(0)}+\frac{a M_{1}}{f(0)}, \beta_{2} \geq b+d$, and define $H=$ $\left(H_{1}, H_{2}\right): C\left(\mathbb{R}, \mathbb{R}^{2}\right) \rightarrow C\left(\mathbb{R}, \mathbb{R}^{2}\right)$ by

$$
H_{i}\left(\phi_{1}, \phi_{2}\right)(t)=h_{i}\left(\phi_{1}, \phi_{2}\right)(t)+\beta_{i} \phi_{i}(t), \quad i=1,2, \forall\left(\phi_{1}(t), \phi_{2}(t)\right) \in C\left(\mathbb{R}, \mathbb{R}^{2}\right) .
$$

Set $C_{[0, M]}\left(\mathbb{R}, \mathbb{R}^{2}\right)=\left\{\varphi \in C\left(\mathbb{R}, \mathbb{R}^{2}\right), 0 \leq \varphi(t) \leq M, \forall t \in \mathbb{R}\right\}$ and $M=\left\{M_{1}, M_{2}\right\}$. Then system (4.1) can be rewritten as

$$
\begin{aligned}
& d_{1} \phi_{1}^{\prime \prime}(t)-c \phi_{1}^{\prime}(t)-\beta_{1} \phi_{1}(t)+H_{1}\left(\phi_{1}, \phi_{2}\right)(t)=0, \\
& d_{2} \phi_{2}^{\prime \prime}(t)-c \phi_{2}^{\prime}(t)-\beta_{2} \phi_{2}(t)+H_{2}\left(\phi_{1}, \phi_{2}\right)(t)=0 .
\end{aligned}
$$


Define

$$
\begin{array}{ll}
\lambda_{11}=\frac{c-\sqrt{c^{2}+4 \beta_{1} d_{1}}}{2 d_{1}}, & \lambda_{12}=\frac{c+\sqrt{c^{2}+4 \beta_{1} d_{1}}}{2 d_{1}}, \\
\lambda_{21}=\frac{c-\sqrt{c^{2}+4 \beta_{2} d_{2}}}{2 d_{2}}, & \lambda_{22}=\frac{c+\sqrt{c^{2}+4 \beta_{2} d_{2}}}{2 d_{2}} .
\end{array}
$$

Define $F=\left(F_{1}, F_{2}\right): C_{[0, M]}\left(\mathbb{R}, \mathbb{R}^{2}\right) \rightarrow C\left(\mathbb{R}, \mathbb{R}^{2}\right)$ by

$$
F_{i}\left(\phi_{1}, \phi_{2}\right)(t)=\frac{1}{d_{i}\left(\lambda_{i 2}-\lambda_{i 1}\right)}\left[\int_{-\infty}^{t} e^{\lambda_{i 1}(t-s)} H_{i}\left(\phi_{1}, \phi_{2}\right)(s) \mathrm{d} s+\int_{t}^{\infty} e^{\lambda_{i 2}(t-s)} H_{i}\left(\phi_{1}, \phi_{2}\right)(s) \mathrm{d} s\right],
$$

$i=1,2$. Then $F_{i}\left(\phi_{1}, \phi_{2}\right)$ satisfies

$$
d_{i} F_{i}^{\prime \prime}(\phi)-c F_{i}^{\prime}(\phi)-\beta_{i} F_{i}(\phi)+H_{i}(\phi)=0, \quad i=1,2 .
$$

Let $\xi>0$ and $\xi<\min \left\{-\lambda_{11},-\lambda_{21}\right\}$. Denote $B_{\xi}\left(\mathbb{R}, \mathbb{R}^{2}\right)=\left\{\phi \in C_{[0, M]}\left(\mathbb{R}, \mathbb{R}^{2}\right):|\phi|_{\xi}<\infty\right\}$, where

$$
|\phi|_{\xi}=\sup _{t \in \mathbb{R}}|\phi(t)| e^{-\xi|t|}
$$

with $|\cdot|$ denoting the maximum norm in $\mathbb{R}^{2}$. It is easy to prove that $B_{\xi}\left(\mathbb{R}, \mathbb{R}^{2}\right)$ is a Banach space.

Lemma 4.3 If $\left(\mathrm{A}_{3}\right)$ holds, then

(1) $H_{1}\left(\omega_{1}, \phi_{2}\right) \leq H_{1}\left(\phi_{1}, \phi_{2}\right) \leq H_{1}\left(\phi_{1}, \omega_{2}\right)$,

(2) $H_{2}\left(\omega_{1}, \omega_{2}\right) \leq H_{2}\left(\phi_{1}, \phi_{2}\right)$,

where $t \in \mathbb{R},\left(\omega_{1}, \omega_{2}\right),\left(\phi_{1}, \phi_{2}\right) \in C_{[0, M]}\left(\mathbb{R}, \mathbb{R}^{2}\right), 0 \leq \omega_{i} \leq \phi_{i} \leq M_{i}, i=1,2$.

Proof The proof can given by direct calculations, so we omit it.

From Lemma 4.3 it is easy to obtain the following conclusion.

Lemma 4.4 If $\left(\mathrm{A}_{3}\right)$ holds, then

(1) $F_{1}\left(\omega_{1}, \phi_{2}\right) \leq F_{1}\left(\phi_{1}, \phi_{2}\right) \leq F_{1}\left(\phi_{1}, \omega_{2}\right)$,

(2) $F_{2}\left(\omega_{1}, \omega_{2}\right) \leq F_{2}\left(\phi_{1}, \phi_{2}\right)$,

where $t \in \mathbb{R},\left(\omega_{1}, \omega_{2}\right),\left(\phi_{1}, \phi_{2}\right) \in C_{[0, M]}\left(\mathbb{R}, \mathbb{R}^{2}\right), 0 \leq \omega_{i} \leq \phi_{i} \leq M_{i}, i=1,2$.

Lemma 4.5 The mapping $F=\left(F_{1}, F_{2}\right): C_{[0, M]}\left(\mathbb{R}, \mathbb{R}^{2}\right) \rightarrow C\left(\mathbb{R}, \mathbb{R}^{2}\right)$ is continuous with respect to the norm $|\cdot|_{\xi}$ in $B_{\xi}\left(\mathbb{R}, \mathbb{R}^{2}\right)$.

Proof First, we prove that $H=\left(H_{1}, H_{2}\right): C_{[0, M]}\left(\mathbb{R}, \mathbb{R}^{2}\right) \rightarrow C\left(\mathbb{R}, \mathbb{R}^{2}\right)$ is continuous with respect to the norm $|\cdot|_{\xi}$ in $B_{\xi}\left(\mathbb{R}, \mathbb{R}^{2}\right)$.

For any $\omega=\left(\omega_{1}, \omega_{2}\right)$ and $\varphi=\left(\varphi_{1}, \varphi_{2}\right) \in C_{[0, M]}\left(\mathbb{R}, \mathbb{R}^{2}\right)$ that satisfy $|\omega-\varphi|_{\xi}=\sup _{t \in \mathbb{R}} \mid \omega(t)-$ $\varphi(t) \mid e^{-\xi|t|}<\delta$, we have

$$
\begin{aligned}
& \left|H_{1}\left(\omega_{1}, \omega_{2}\right)(t)-H_{1}\left(\varphi_{1}, \varphi_{2}\right)(t)\right| e^{-\xi|t|} \\
& \quad=\left|h_{1}\left(\omega_{1}, \omega_{2}\right)(t)+\beta_{1} \omega_{1}-h_{1}\left(\varphi_{1}, \varphi_{2}\right)(t)-\beta \varphi_{1}\right| e^{-\xi|t|}
\end{aligned}
$$




$$
\begin{aligned}
&=\left|\frac{a \omega_{1}}{f\left(\omega_{1}\right)}\left(N-\omega_{1}-\omega_{2}\right)-\frac{a \varphi_{1}}{f\left(\varphi_{1}\right)}\left(N-\varphi_{1}-\varphi_{2}\right)+\left(\beta_{1}-(d+\gamma)\right)\left(\omega_{1}-\varphi_{1}\right)\right| e^{-\xi|t|} \\
&= \mid a \frac{\left(\omega_{1} f\left(\varphi_{1}\right)-\varphi_{1} f\left(\omega_{1}\right)\right)\left(N-\varphi_{1}-\varphi_{2}\right)}{f\left(\varphi_{1}\right) f\left(\omega_{1}\right)}-\frac{a \omega_{1}\left(\omega_{1}-\varphi_{1}\right)}{f\left(\omega_{1}\right)}-\frac{a \omega_{1}\left(\omega_{2}-\varphi_{2}\right)}{f\left(\omega_{1}\right)} \\
&+\left(\beta_{1}-d-\gamma\right)\left(\omega_{1}-\varphi_{1}\right) \mid e^{-\xi|t|} \\
& \leq {\left[a N \frac{\left|\omega_{1}-\varphi_{1}\right|}{f\left(\omega_{1}\right)}+a N \frac{\varphi_{1}\left|f\left(\varphi_{1}\right)-f\left(\omega_{1}\right)\right|}{f\left(\omega_{1}\right) f\left(\varphi_{1}\right)}+\left(\frac{a M_{1}}{f(0)}+\beta_{1}-d-\gamma\right)\left|\omega_{1}-\varphi_{1}\right|\right.} \\
&\left.+\frac{a M_{1}}{f(0)}\left|\omega_{2}-\varphi_{2}\right|\right] e^{-\xi|t|} \\
& \leq {\left[\frac{a N}{f(0)}\left|\omega_{1}-\varphi_{1}\right|+\frac{a N M_{1} L}{f^{2}(0)}\left|\omega_{1}-\varphi_{1}\right|+\left(\frac{a M_{1}}{f(0)}+\beta_{1}-d-\gamma\right)\left|\omega_{1}-\varphi_{1}\right|\right.} \\
&\left.+\frac{a M_{1}}{f(0)}\left|\omega_{2}-\varphi_{2}\right|\right] e^{-\xi|t|} \\
& \leq\left(\frac{a N}{f(0)}+\frac{a N M_{1} L}{f^{2}(0)}+2 \frac{a M_{1}}{f(0)}+\beta_{1}-d-\gamma\right)|\omega(t)-\varphi(t)|_{\xi}, \\
&\left|H_{2}\left(\omega_{1}, \omega_{2}\right)(t)-H_{2}\left(\varphi_{1}, \varphi_{2}\right)(t)\right| e^{-\xi|t|} \\
&=\left|h_{2}\left(\omega_{1}, \omega_{2}\right)(t)+\beta_{2} \omega_{2}-h_{2}\left(\varphi_{1}, \varphi_{2}\right)(t)-\beta_{2} \varphi_{1}\right| e^{-\xi|t|} \\
& \leq {\left[\gamma\left|\omega_{1}-\varphi_{1}\right|+\left(\beta_{2}-(b+d)\right)\left|\omega_{2}-\varphi_{2}\right|\right] e^{-\xi|t|} } \\
& \leq\left(\gamma+\beta_{2}-(b+d)\right)|\omega(t)-\varphi(t)| \xi .
\end{aligned}
$$
Now, for any $\varepsilon>0$, choose $\delta=\min \left\{\frac{\frac{a N}{f(0)} \frac{a N M_{1} L}{f^{2}(0)}+2 \frac{a M_{1}}{f(0)}+\beta_{1}-d-\gamma}{\gamma+\beta_{2}-(b+d)}\right\}$. If $|\omega(t)-\varphi(t)|_{\xi}<\delta$,
then we have

$$
\left|H_{i}\left(\omega_{1}, \omega_{2}\right)(t)-H_{i}\left(\varphi_{1}, \varphi_{2}\right)(t)\right| e^{-\xi|t|}<\varepsilon, \quad i=1,2 .
$$

So $H$ is continuous with respect to the norm $|\cdot|_{\xi}$ in $B_{\xi}\left(\mathbb{R}, \mathbb{R}^{2}\right)$.

Next, we prove that $F=\left(F_{1}, F_{2}\right): C_{[0, M]}\left(\mathbb{R}, \mathbb{R}^{2}\right) \rightarrow C\left(\mathbb{R}, \mathbb{R}^{2}\right)$ is continuous with respect to the norm $|\cdot|_{\xi}$ in $B_{\xi}\left(\mathbb{R}, \mathbb{R}^{2}\right)$. When $t \geq 0$,

$$
\begin{aligned}
\left|F_{1}\left(\omega_{1}, \omega_{2}\right)(t)-F_{1}\left(\varphi_{1}, \varphi_{2}\right)(t)\right| e^{-\xi|t|} & \mid \frac{1}{d_{1}\left(\lambda_{12}-\lambda_{11}\right)}\left[\int_{-\infty}^{t} e^{\lambda_{11}(t-s)}\left(H_{1}\left(\omega_{1}, \omega_{2}\right)-H_{1}\left(\varphi_{1}, \varphi_{2}\right)\right) \mathrm{d} s\right. \\
& \left.+\int_{t}^{\infty} e^{\lambda_{12}(t-s)}\left(H_{1}\left(\omega_{1}, \omega_{2}\right)-H_{1}\left(\varphi_{1}, \varphi_{2}\right)\right) \mathrm{d} s\right] \mid e^{-\xi|t|} \\
\leq & \frac{1}{d_{1}\left(\lambda_{12}-\lambda_{11}\right)}\left[\int_{-\infty}^{t} e^{\lambda_{11}(t-s)}\left|H_{1}\left(\omega_{1}, \omega_{2}\right)-H_{1}\left(\varphi_{1}, \varphi_{2}\right)\right| \mathrm{d} s\right. \\
& \left.+\int_{t}^{\infty} e^{\lambda_{12}(t-s)}\left|H_{1}\left(\omega_{1}, \omega_{2}\right)-H_{1}\left(\varphi_{1}, \varphi_{2}\right)\right| \mathrm{d} s\right] e^{-\xi|t|} \\
= & \frac{1}{d_{1}\left(\lambda_{12}-\lambda_{11}\right)}\left[\int_{-\infty}^{0} e^{\lambda_{11}(t-s)} e^{\xi|s|}\left|H_{1}\left(\omega_{1}, \omega_{2}\right)-H_{1}\left(\varphi_{1}, \varphi_{2}\right)\right| e^{-\xi|s|} \mathrm{d} s\right. \\
& +\int_{0}^{t} e^{\lambda_{11}(t-s)} e^{\xi|s|}\left|H_{1}\left(\omega_{1}, \omega_{2}\right)-H_{1}\left(\varphi_{1}, \varphi_{2}\right)\right| e^{-\xi|s|} \mathrm{d} s
\end{aligned}
$$




$$
\begin{aligned}
& \left.+\int_{t}^{\infty} e^{\lambda_{12}(t-s)} e^{\xi|s|}\left|H_{1}\left(\omega_{1}, \omega_{2}\right)-H_{1}\left(\varphi_{1}, \varphi_{2}\right)\right| e^{-\xi|s|} \mathrm{d} s\right] e^{-\xi|t|} \\
\leq & \frac{1}{d_{1}\left(\lambda_{12}-\lambda_{11}\right)}\left[\int_{-\infty}^{0} e^{\lambda_{11}(t-s)} e^{-\xi s} \mathrm{~d} s+\int_{0}^{t} e^{\lambda_{11}(t-s)} e^{\xi s} \mathrm{~d} s+\int_{t}^{\infty} e^{\lambda_{12}(t-s)} e^{\xi s} \mathrm{~d} s\right] \\
& \cdot\left|H_{1}\left(\omega_{1}, \omega_{2}\right)-H_{1}\left(\varphi_{1}, \varphi_{2}\right)\right|_{\xi} e^{-\xi|t|} \\
= & \frac{1}{d_{1}\left(\lambda_{12}-\lambda_{11}\right)}\left[\frac{\left(\lambda_{12}-\lambda_{11}\right) e^{\xi t}}{\left(\xi-\lambda_{11}\right)\left(\lambda_{12}-\xi\right)}+\frac{2 \xi e^{\lambda_{11} t}}{\lambda_{11}^{2}-\xi^{2}}\right] e^{-\xi t}\left|H_{1}\left(\omega_{1}, \omega_{2}\right)-H_{1}\left(\varphi_{1}, \varphi_{2}\right)\right|_{\xi} \\
\leq & \frac{1}{d_{1}\left(\lambda_{12}-\lambda_{11}\right)}\left[\frac{\lambda_{12}-\lambda_{11}}{\left(\xi-\lambda_{11}\right)\left(\lambda_{12}-\xi\right)}+\frac{2 \xi}{\lambda_{11}^{2}-\xi^{2}}\right]\left|H_{1}\left(\omega_{1}, \omega_{2}\right)-H_{1}\left(\varphi_{1}, \varphi_{2}\right)\right|_{\xi} .
\end{aligned}
$$

When $t<0$,

$$
\begin{aligned}
& \left|F_{1}\left(\omega_{1}, \omega_{2}\right)(t)-F_{1}\left(\varphi_{1}, \varphi_{2}\right)(t)\right| e^{-\xi|t|} \\
& \quad \leq \frac{1}{d_{1}\left(\lambda_{12}-\lambda_{11}\right)}\left[\frac{\lambda_{12}-\lambda_{11}}{-\left(\xi+\lambda_{11}\right)\left(\lambda_{12}+\xi\right)}+\frac{2 \xi}{\lambda_{12}^{2}-\xi^{2}}\right]\left|H_{1}\left(\omega_{1}, \omega_{2}\right)-H_{1}\left(\varphi_{1}, \varphi_{2}\right)\right|_{\xi} .
\end{aligned}
$$

Hence, $F_{1}$ is continuous with respect to the norm $|\cdot|_{\xi}$ in $B_{\xi}\left(\mathbb{R}, \mathbb{R}^{2}\right)$. Using similar arguments, we can show that $F_{2}$ is continuous with respect to the norm $|\cdot|_{\xi}$ in $B_{\xi}\left(\mathbb{R}, \mathbb{R}^{2}\right)$. Then $F$ is continuous with respect to the norm $|\cdot|_{\xi}$ in $B_{\xi}\left(\mathbb{R}, \mathbb{R}^{2}\right)$.

Define the set

$$
\Gamma=\left\{\phi \in C_{[0, M]}\left(\mathbb{R}, \mathbb{R}^{2}\right): \underline{\phi} \leq \phi \leq \bar{\phi}\right\}
$$

where $\underline{\phi}$ and $\bar{\phi}$ are a pair of upper and lower solutions satisfying $\left(\mathrm{B}_{1}\right),\left(\mathrm{B}_{2}\right),\left(\mathrm{B}_{3}\right)$. Obviously, $\Gamma$ is nonempty. Moreover, it is easy to check that $\Gamma$ is a closed, bounded, and convex subset of $B_{\xi}\left(\mathbb{R}, \mathbb{R}^{2}\right)$.

Lemma 4.6 The operator $F=\left(F_{1}, F_{2}\right)$ maps $\Gamma$ into $\Gamma$.

Proof For any $\left(\underline{\phi}_{1}, \underline{\phi}_{2}\right) \leq\left(\phi_{1}, \phi_{2}\right) \leq\left(\bar{\phi}_{1}, \bar{\phi}_{2}\right)$, it follows from Lemma 4.4 that

$$
\begin{aligned}
& F_{1}\left(\underline{\phi}_{1}, \bar{\phi}_{2}\right) \leq F_{1}\left(\underline{\phi}_{1}, \phi_{2}\right) \leq F_{1}\left(\phi_{1}, \phi_{2}\right) \leq F_{1}\left(\phi_{1}, \underline{\phi}_{2}\right) \leq F_{1}\left(\bar{\phi}_{1}, \underline{\phi}_{2}\right), \\
& F_{2}\left(\underline{\phi}_{1}, \underline{\phi_{2}}\right) \leq F_{2}\left(\phi_{1}, \phi_{2}\right) \leq F_{1}\left(\bar{\phi}_{1}, \bar{\phi}_{2}\right) .
\end{aligned}
$$

So, it suffices to prove that

$$
\begin{array}{ll}
F_{1}\left(\underline{\phi}_{1}, \bar{\phi}_{2}\right) \geq \underline{\phi}_{1}, \quad F_{1}\left(\bar{\phi}_{1}, \underline{\phi}_{2}\right) \leq \bar{\phi}_{1}, \\
F_{2}\left(\underline{\phi}_{1}, \underline{\phi}_{2}\right) \geq \underline{\phi}_{2}, \quad F_{2}\left(\bar{\phi}_{1}, \bar{\phi}_{2}\right) \leq \bar{\phi}_{2} .
\end{array}
$$

In fact,

$$
\begin{aligned}
& F_{1}\left(\bar{\phi}_{1}, \underline{\phi}_{2}\right) \\
& \quad=\frac{1}{d_{1}\left(\lambda_{12}-\lambda_{11}\right)}\left[\int_{-\infty}^{t} e^{\lambda_{11}(t-s)} H_{1}\left(\bar{\phi}_{1}, \underline{\phi}_{2}\right)(s) \mathrm{d} s+\int_{t}^{\infty} e^{\lambda_{12}(t-s)} H_{1}\left(\bar{\phi}_{1}, \underline{\phi}_{2}\right)(s) \mathrm{d} s\right]
\end{aligned}
$$




$$
\begin{aligned}
& =\frac{1}{d_{1}\left(\lambda_{12}-\lambda_{11}\right)}\left[\int_{-\infty}^{t} e^{\lambda_{11}(t-s)}+\int_{t}^{\infty} e^{\lambda_{12}(t-s)}\right] H_{1}\left(\bar{\phi}_{1}, \underline{\phi}_{2}\right)(s) \mathrm{d} s \\
& \leq \frac{1}{d_{1}\left(\lambda_{12}-\lambda_{11}\right)}\left[\int_{-\infty}^{t} e^{\lambda_{11}(t-s)}+\int_{t}^{\infty} e^{\lambda_{12}(t-s)}\right]\left[-\bar{\phi}^{\prime \prime}(t)+c \bar{\phi}_{1}^{\prime}(t)+\beta_{1} \bar{\phi}_{1}\right] \mathrm{d} s \\
& =\bar{\phi}_{1}(t)+\frac{1}{d_{1}\left(\lambda_{12}-\lambda_{11}\right)}\left(\sum_{j=1}^{k} e^{\lambda_{11}\left(t-t_{j}\right)}+\sum_{j=k+1}^{m} e^{\lambda_{12}\left(t-t_{j}\right)}\right)\left(\bar{\phi}_{1}^{\prime}\left(t_{j}^{+}\right)-\bar{\phi}_{1}^{\prime}\left(t_{j}^{-}\right)\right) \\
& \leq \bar{\phi}_{1}(t) .
\end{aligned}
$$

Furthermore, since $F_{1}\left(\bar{\phi}_{1}, \underline{\phi}_{2}\right)(t)$ and $\bar{\phi}_{1}(t)$ are both continuous for $t \in \mathbb{R}$, we could take $t \rightarrow t_{k}^{+}$in the inequality into $F_{1}\left(\bar{\phi}_{1}, \underline{\phi}_{2}\right)\left(t_{k}^{+}\right)$for all $t_{k}^{+} \in \Sigma$. Therefore, $F_{1}\left(\bar{\phi}_{1}, \underline{\phi}_{2}\right) \leq \bar{\phi}_{1}$ for all $t \in \mathbb{R}$. Using similar arguments, we can obtain that $F_{1}\left(\bar{\phi}_{1}, \underline{\phi}_{2}\right) \leq \bar{\phi}_{1}, F_{2}\left(\underline{\phi}_{1}, \underline{\phi}_{2}\right) \geq \underline{\phi}_{2}$, $F_{2}\left(\bar{\phi}_{1}, \bar{\phi}_{2}\right) \leq \bar{\phi}_{2}$ in $\mathbb{R}$.

Lemma 4.7 The operator $F=\left(F_{1}, F_{2}\right): \Gamma \rightarrow \Gamma$ is compact with respect to the norm $|\cdot|_{\xi}$ in $B_{\xi}\left(\mathbb{R}, \mathbb{R}^{2}\right)$.

Proof The proof of Lemma 4.7 is similar to that of [24], Lemma 3.6, so we also omit the proof.

Combining Lemmas 4.5-4.7 with the Schauder fixed point theorem, we get that there exists a fixed point $\phi=\left(\phi_{1}, \phi_{2}\right)$ of $F$ that is a solution of system (4.1), that is, $F\left(\phi_{1}, \phi_{2}\right)=$ $\left(\phi_{1}, \phi_{2}\right)$.

Next, we verify the boundary conditions (4.2). Since $\left(\phi_{1}, \phi_{2}\right) \subset \Gamma$, we have

$$
(0,0) \leq\left(\underline{\phi}_{1}, \underline{\phi}_{2}\right) \leq\left(\phi_{1}, \phi_{2}\right) \leq\left(\bar{\phi}_{1}, \bar{\phi}_{2}\right) \leq\left(M_{1}, M_{2}\right) \text {. }
$$

Hence, by $\left(B_{2}\right)$ we obtain

$$
\lim _{t \rightarrow-\infty}\left(\phi_{1}(t), \phi_{2}(t)\right)=(0,0), \quad \lim _{t \rightarrow \infty}\left(\phi_{1}(t), \phi_{2}(t)\right)=\left(I^{*}, R^{*}\right) .
$$

So the fixed point $\left(\phi_{1}, \phi_{2}\right)$ satisfies the asymptotic boundary conditions (4.2). Thus, $\phi=$ $\left(\phi_{1}(t), \phi_{2}(t)\right)$ is a traveling wave solution of system (4.1). Then we immediately have the following conclusion.

Theorem 4.1 If system (4.1) has a pair of upper and lower solutions $\bar{\phi}=\left(\bar{\phi}_{1}, \bar{\phi}_{2}\right), \underline{\phi}=$ $\left(\underline{\phi}_{1}, \underline{\phi}_{2}\right)$ satisfying $\left(\mathrm{B}_{1}\right),\left(\mathrm{B}_{2}\right),\left(\mathrm{B}_{3}\right)$, then system $(4.1)$ admits a traveling wave solution connecting $E_{0}=(0,0)$ and $E^{*}=\left(I^{*}, R^{*}\right)$.

By Lemmas 4.1 and 4.2 and Theorem 4.1 we immediately obtain the following conclusion.

Theorem 4.2 Let $1<R_{0}<1+\frac{b+d}{\gamma}$. Then, for every $c>c^{*}$, there exists a traveling wave solution of system $(1.3)$ with speed c connecting $(0,0)$ and $\left(I^{*}, R^{*}\right)$.

\section{Sensitivity analysis}

To illustrate the theoretical results obtained in this paper, we give some numerical simulations. For simplicity, we take the function $f(I)$ of the form $1+\beta I$. It is easy to prove that this 
function satisfies conditions $\left(A_{1}\right)-\left(A_{3}\right)$. Consider system (1.3) under the Neumann boundary conditions as follows:

$$
\frac{\partial I}{\partial n}=\frac{\partial R}{\partial n}=0, \quad t>0, x=0,20 .
$$

Fix the parameter values $a=0.6, \beta=0.5, d=0.1, \gamma=0.2, b=0.1$ and change the population size $N$.

Example 5.1 Fix $N=0.4$. Then $R_{0}=0.8<1$. Hence, system (1.3) has only a stable diseasefree equilibrium $E_{0}=(0,0)$; see Figure 1 .

Example 5.2 Fix $N=5$. Then $R_{0}=10>1$. Hence, system (1.3) has only a stable disease equilibrium $E^{*}=(1.6619,1.6619)$; see Figure 2 .

In order to illustrate the existence of travelling wave solution, we consider the following piecewise functions as initial conditions:

$$
(I(0, x), R(0, x))= \begin{cases}E^{*}, & x \in[-20,0), \\ E^{0}, & x \in[0,20],\end{cases}
$$

where $E_{0}=(0,0)$, and $E^{*}$ is as in Lemma 2.1 .
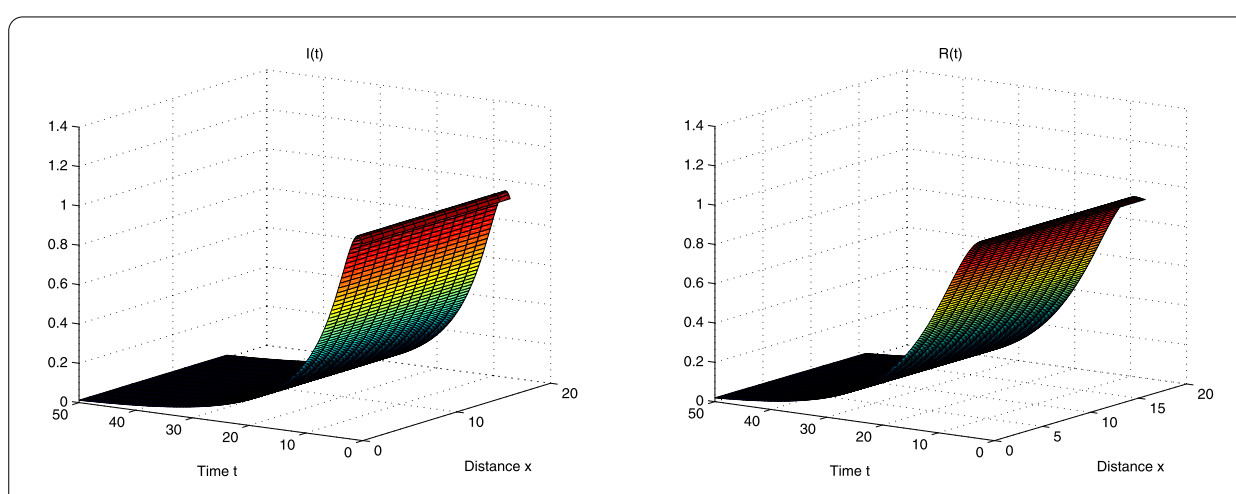

Figure 1 Numerical simulations of system (1.3) with parameters of Example 5.1 and initial conditions $\boldsymbol{I}(\mathbf{0}, \boldsymbol{x})=\boldsymbol{R}(\mathbf{0}, \boldsymbol{x})=\mathbf{1 . 6}$. The disease-free steady state is globally asymptotically stable.

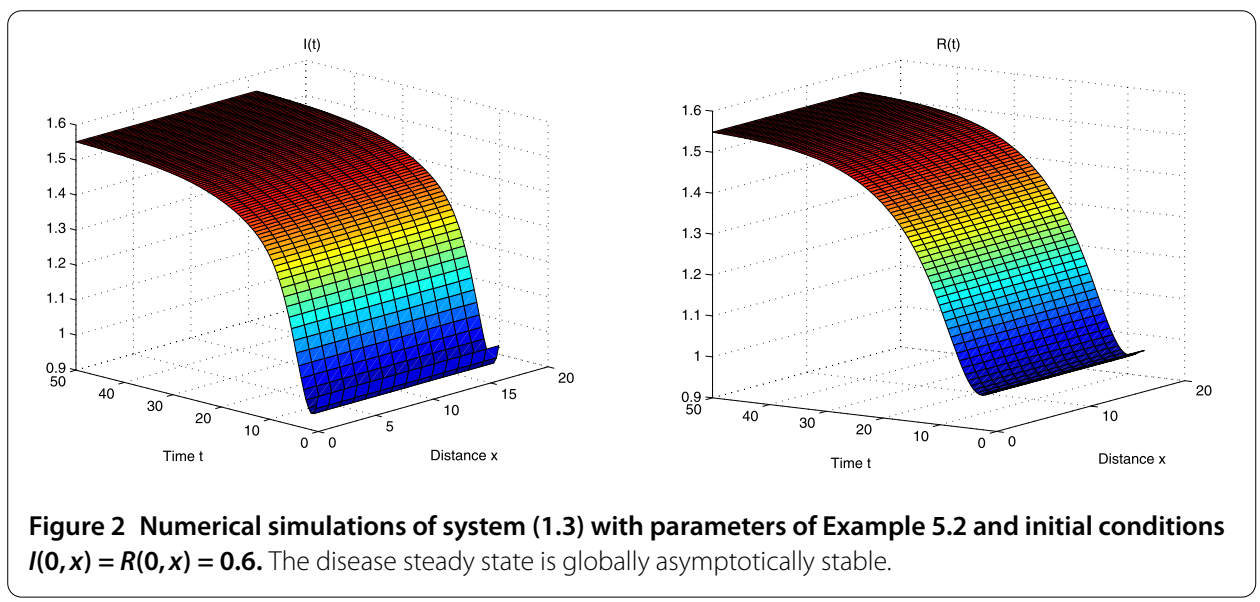



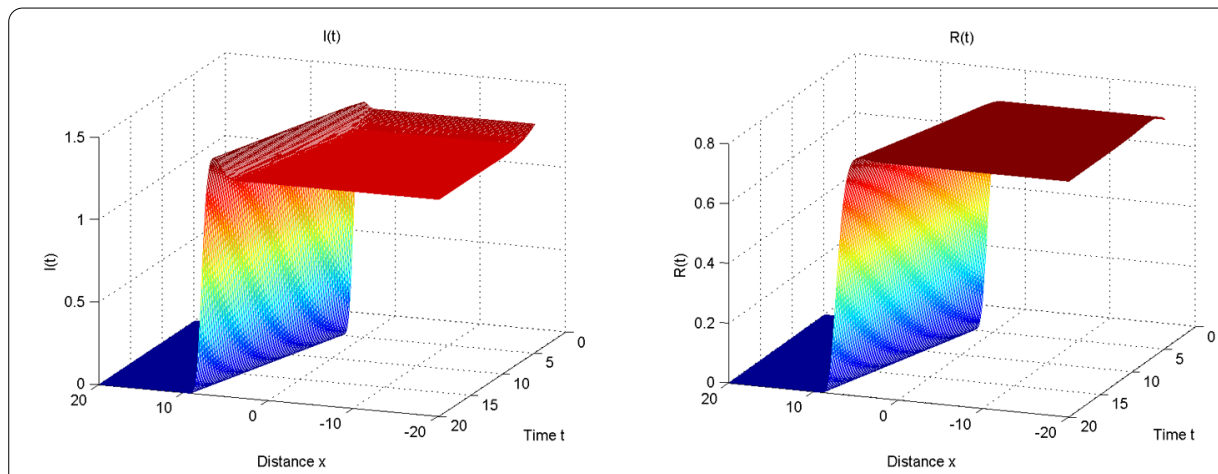

Figure 3 The travelling wave observed in system (1.3) with parameters $d_{1}=0.04, d_{2}=0.02, a=0.8$, $N=2.1, d=0.4, \gamma=0.3, b=0.1, \beta=0.5$.

Example 5.3 Set $d_{1}=0.04, d_{2}=0.02, a=0.8, N=2.1, d=0.4, \gamma=0.3, b=0.1, \beta=0.5$. Then $1<R_{0}=2.4<1+\frac{b+d}{\gamma}=2.667$. It follows from Theorem 4.2 that system (1.3) exists a travelling wave solution with speed $c>c^{*}$ connecting $(0,0)$ and $(0.6,0.36)$; see Figure 3.

\section{Summary and discussion}

The basic reproduction number $R_{0}$ is the threshold of whether an endemic disease persists or not. In this paper, an SIRS model with diffusion is studied. We make a more general assumption that the infection force is $\frac{a I}{f(I)}$, which is satisfied with $\left(\mathrm{A}_{1}\right)-\left(\mathrm{A}_{3}\right)$, and then our conclusions are fit for a wider range. The existence of equilibria and their local stability and global stability are established. We show that the disease-free equilibrium $E_{0}$ is locally asymptotically stable when $R_{0}<1$, whereas the endemic steady state $E^{*}$ is local asymptotically stable when $R_{0}>1$. Furthermore, the $E_{0}$ is globally asymptotically stable if $R_{0} \leq 1$, that is, the disease always dies out eventually from the biological point of view. The state $E^{*}$ is globally asymptotically stable if $R_{0}>1$, which implies that the disease will persist. According to the stability results, we can design the intervention strategies to guard against some endemic diseases. In the case of $R_{0}>1$, some corresponding parameters are changed so that the $R_{0}$ is below one.

Furthermore, combining the technique of upper-lower solutions with the Schauder fixed point theorem, we obtain the existence of traveling wave solution of system (1.3) connecting the disease free steady state $E_{0}$ and the endemic steady state $E^{*}$, provided that $1<R_{0}<1+\frac{b+d}{\gamma}$ and the spatial velocity of infection $c$ is greater than the minimal wave speed $c^{*}=\max \left\{2 \sqrt{d_{1} D}, 2 \sqrt{d_{2} D}\right\}$, which depends on the diffusion parameters $d_{1}$ and $d_{2}$. The conclusion indicates that when epidemic spreading happens, the spatial velocity of infection can be influenced by diffusion of infectious and recovered individuals. These theoretical results may help to provide some prediction and prevention guide of infectious diseases. 


\section{Acknowledgements}

This research has been partially supported by the NSF of China (No. 61373174), the PhD Fellowship of Xidian University (No. 20103146145), and Chinese Universities Scientific Fund (No. 20101156145). The authors are very grateful to the editor and the anonymous referees for their valuable comments and suggestions, which greatly improved the presentation of this work.

Received: 9 July 2016 Accepted: 13 September 2016 Published online: 29 September 2016

\section{References}

1. Kermack, WO, McKendrick, AG: A contribution to the mathematical theory of epidemics. Proc. R. Soc. Lond. Ser. A 115, 700-721 (1927)

2. Earn, DJD, Rohani, P, Bolker, BM, Grenfell, BT: A simple model for complex dynamical transitions in epidemics. Science 287, 667-670 (2000)

3. Diekmann, O, Kreztschmar, M: Patterns in the effects of infectious disease on population growth. J. Math. Biol. 29, 539-570 (1991)

4. Anderson, RM, May, RM: Infectious Diseases of Humans: Dynamics and Control. Oxford University Press, Oxford (1992)

5. Capasso, V: Mathematical Structures of Epidemic Systems. Springer, Heidelberg (1993)

6. Brauer, F, van den Driessche, P: Models for translation of disease with immigration of infectives. Math. Biosci. 171, 143-154 (2001)

7. Shan, C, Zhu, H: Bifurcations and complex dynamics of an SIR model with the impact of the number of hospital beds. J. Differ. Equ. 257, 1662-1688 (2014)

8. Hethcote, HW: The mathematics of infectious disease. SIAM Rev. 42, 599-653 (2000)

9. Mena-Lorca, J, Hethcote, HW: Dynamic models of infectious disease as regulators of population sizes. J. Math. Biol. 30, 693-716 (1992)

10. Wang, W, Zhao, X: An endemic model in a patchy environment. Math. Biosci. 190, 97-112 (2004)

11. Hethcote, HW, Levin, SA: Periodicity in epidemiological models. In: Applied Mathematical Ecology (Trieste, 1986). Biomathematics, vol. 18, pp. 193-211. Springer, Berlin (1989)

12. Alexander, ME, Moghadas, SM: Periodicity in an epidemic model with a generalized non-linear incidence. Math. Biosci. 189, 75-96 (2004)

13. Derrick, WR, van den Driessche, P: Homoclinic orbits in a disease transmission model with nonlinear incidence and nonconstant population. Discrete Contin. Dyn. Syst., Ser. B 3, 299-309 (2003)

14. Xiao, D, Ruan, S: Global analysis of an epidemic model with nonmonotone incidence rate. Math. Biosci. 208, 419-429 (2007)

15. Ruan, S, Wang, W: Dynamical behavior of an epidemic model with a nonlinear incidence rate. J. Differ. Equ. 188 135-163 (2003)

16. Capasso, V, Serio, G: A generalization of the Kermack-McKendrick deterministic epidemic model. Math. Biosci. 42 41-61 (1978)

17. Liu, W, Levin, SA, Iwasa, Y: Influence of nonlinear incidence rates upon the behavior of SIRS epidemiological models. J. Math. Biol. 23, 187-204 (1986)

18. Liu, W, Hethcote, HW, Levin, SA: Dynamical behavior of epidemiological models with nonlinear incidence rates. J. Math. Biol. 25, 359-380 (1987)

19. Wang, W: Epidemic models with nonlinear infection forces. Math. Biosci. Eng. 3, 267-279 (2006)

20. van den Driessche, P, Watmough, J: A simple SIS epidemic model with a backward bifurcation. J. Math. Biol. 40, 525-540 (2000)

21. van den Driessche, P, Watmough, J: Epidemic solutions and endemic catastrophes. Fields Inst. Commun. 36, 247-257 (2003)

22. Derrick, WR, van den Driessche, P: A disease transmission model in a nonconstant population. J. Math. Biol. 31 , 495-512 (1993)

23. $\mathrm{Hu}, \mathrm{Z}, \mathrm{Bi}, \mathrm{P}, \mathrm{Ma}, \mathrm{W}$, Ruan, S: Bifurcations of an SIRS epidemic model with nonlinear incidence rate. Discrete Contin. Dyn. Syst., Ser. B 1, 93-112 (2011)

24. Li, W, Lin, G, Ruan, S: Existence of traveling wave solutions in delayed reaction-diffusion systems with applications to diffusion-competition systems. Nonlinearity 19, 1253-1257 (2006)

25. Wang, Z, Wu, J, Liu, R: Traveling waves of the spread of avian influenza. Proc. Am. Math. Soc. 140(11), 3931-3946 (2012)

26. Wang, Z, Li, W, Ruan, S: Traveling wave fronts in reaction-diffusion systems with spatiotemporal delays. J. Differ. Equ. $222,185-232(2006)$

27. Xu, Z, Zhao, Y: A reaction diffusion model of dengue transmission. Discrete Contin. Dyn. Syst., Ser. B 9, 2993-3018 $(2014)$

28. Wang, W, Zhao, X: A nonlocal and time-delayed reaction-diffusion model of dengue transmission. SIAM J. Appl. Math 71, 147-168 (2011)

29. Martin, R, Smith, H: Abstract functional differential and reaction-diffusion systems. Trans. Am. Math. Soc. 321, 1-44 (1990)

30. Xu, R, Ma, Z: An HBV model with diffusion and time delay. J. Theor. Biol. 257, 449-509 (2009)

31. Du, Y, Hsu, SH: A diffusive predator-prey model in heterogeneous environment. J. Differ. Equ. 203, 331-364 (2004)

32. Henry, D: Geometric Theory of Semilinear Parabolic Equations. Springer, New York (1981) 